\title{
Bladder cancer detection in urine using DNA methylation markers: a technical and prospective preclinical validation
}

Anouk E. Hentschel ${ }^{1,2}$, Irene J. Beijert ${ }^{1,2}$, Judith Bosschieter ${ }^{1}$, Paul C. Kauer ${ }^{3}$, André N. Vis ${ }^{1}$, Birgit I. Lissenberg-Witte ${ }^{4}$, R. Jeroen A. van Moorselaar ${ }^{1}$, Renske D. M. Steenbergen ${ }^{2 \dagger}$ and Jakko A. Nieuwenhuijzen ${ }^{1 * \dagger}$

\begin{abstract}
Background: The development of accurate urinary biomarkers for non-invasive and cost-effective detection of primary and recurrent bladder tumours is recognized as one of the major clinical needs in bladder cancer diagnostics. The purposes of this study were (1) to validate the results of a previous technical comparison by determining the diagnostic performance of nine methylation markers in urine pellet compared to full void urine, and (2) to validate the diagnostic performance of the optimal marker panel GHSR/MAL from a previous exploratory study in a preclinical setting.

Methods: Urine samples of 108 patients with bladder cancer and 100 age- and gender-matched controls were prospectively collected for methylation analysis. Urinary methylation levels of the markers FAM19A4, GHSR, MAL, miR129, miR-935, PHACTR3, PRDM14, SST and ZIC1 were determined with quantitative methylation-specific PCR in urine pellet. Area under the curves (AUCs) were determined for individual markers and the marker panel GHSR/MAL. The diagnostic performance of the marker panel GHSR/MAL was evaluated in the total study population and in different subgroups of patients with bladder cancer using the Chi-square test. The diagnostic accuracy was assessed by leaveone-out cross-validation.

Results: All nine urinary methylation markers (FAM19A4, GHSR, MAL, miR-129, miR-935, PHACTR3, PRDM14, SST and ZIC1) showed significantly higher methylation levels in bladder cancer patients than in controls $(p<0.001)$. Area under the curves (AUCs) of the nine methylation markers tested in urine pellet were similar to AUCs in full void urine of an independent previous cohort. GHSR/MAL reached an AUC of 0.89 (95\% confidence interval [CI] 0.84-0.94), at 80\% sensitivity and $93 \%$ specificity. Sensitivity of GHSR/MAL increased with higher tumour grades, higher tumour stages, in primary vs. recurrent tumours, and in males vs. females.

Conclusions: This technical validation supports the robustness of DNA methylation analysis in urine pellet and full void urine for the non-invasive detection of bladder cancer. Subsequent preclinical validation confirmed the diagnostic potential of GHSR/MAL. These findings underline the diagnostic potential of the marker panel GHSR/MAL for future bladder cancer diagnostics.
\end{abstract}

\footnotetext{
*Correspondence: j.nieuwenhuijzen@amsterdamumc.nl

${ }^{\dagger}$ Renske D. M. Steenbergen, Jakko A. Nieuwenhuijzen: Shared last authorship

${ }^{1}$ Department of Urology, Amsterdam UMC, Vrije Universiteit Amsterdam, Amsterdam, The Netherlands

Full list of author information is available at the end of the article
}

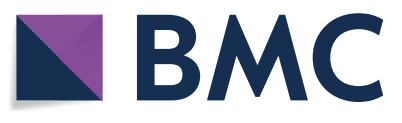

(c) The Author(s) 2022. Open Access This article is licensed under a Creative Commons Attribution 4.0 International License, which permits use, sharing, adaptation, distribution and reproduction in any medium or format, as long as you give appropriate credit to the original author(s) and the source, provide a link to the Creative Commons licence, and indicate if changes were made. The images or other third party material in this article are included in the article's Creative Commons licence, unless indicated otherwise in a credit line to the material. If material is not included in the article's Creative Commons licence and your intended use is not permitted by statutory regulation or exceeds the permitted use, you will need to obtain permission directly from the copyright holder. To view a copy of this licence, visit http://creativecommons.org/licenses/by/4.0/. The Creative Commons Public Domain Dedication waiver (http://creativeco mmons.org/publicdomain/zero/1.0/) applies to the data made available in this article, unless otherwise stated in a credit line to the data. 
Keywords: Biomarkers, Tumour, DNA methylation, Liquid biopsy, Urinary bladder neoplasms, Urine

\section{Introduction}

The presence of a bladder tumour is often discovered after episodes of painless macroscopic haematuria. At initial diagnosis, the disease is non-muscle-invasive in approximately $75 \%$ of patients [1]. Since non-muscleinvasive bladder cancer (NMIBC) has the tendency to recur or progress to muscle-invasive disease, regular and long-term cystoscopic evaluations are mandatory [1]. Yet, cystoscopy has important disadvantages as it is an invasive procedure that is associated with high costs [2]. Urine cytology is used as a complementary and noninvasive tool in patients with high-grade (HG) urothelial bladder tumours, but sensitivity is limited in low-grade (LG) disease [1, 3]. Besides its suboptimal sensitivity, the use of urine cytology is hampered by its subjective interpretation [4]. Therefore, research focuses on the identification of reliable urinary biomarkers to allow for objective, non-invasive and cost-effective detection of bladder cancer [5, 6].

Multiple studies have reported on the potential of DNA methylation markers as urinary biomarkers for bladder cancer diagnostics [7]. DNA hypermethylation in promoter regions of tumour suppressor genes can lead to inactivation of their tumour suppressive function, accordingly contributing to the development of cancer. As DNA hypermethylation is considered to be among the earliest events in urothelial carcinogenesis, DNA methylation analysis poses a promising tool for the early detection of bladder tumours [8]. In a previous exploratory study, DNA methylation analysis in full void urine of patients with bladder cancer $(n=72)$ and healthy controls $(n=75)$ identified ten genes with significantly higher urinary methylation levels in patients with bladder cancer compared to healthy controls [9]. The diagnostic performance proved best for the marker panel GHSR/MAL, reflected by an area under the curve (AUC) of 0.89 , at $92 \%$ sensitivity and $85 \%$ specificity. In a subsequent technical comparison of urine fractions with the nine most discriminative methylation markers, it was demonstrated that urine pellet is preferred over full void urine or urine supernatant [10]. Urine pellet represented the respective tumour tissues best, it reached the highest discriminative capability for the marker panel GHSR/MAL, and it was the most convenient to process.

The aims of the present study are (1) to validate the results of our technical comparison by determining the diagnostic performance of these nine methylation markers (FAM19A4, GHSR, MAL, miR-129, miR-935, PHACTR3, PRDM14, SST and ZIC1) in urine pellet compared to full void urine and (2) to validate the diagnostic performance of the best performing marker panel from our exploratory study (GHSR/MAL) in a preclinical setting.

\section{Materials and methods \\ Patients}

Patients were prospectively included between October 2018 and October 2020 at Amsterdam University Medical Centers and at OLVG. Patients suspected of urothelial carcinoma of the bladder on cystoscopy were eligible for inclusion. Presence of urothelial carcinoma of the bladder had to be confirmed by transurethral resection of the bladder tumour (TURBT). Patients were excluded if no TURBT was performed or if TURBT did not show evidence of urothelial carcinoma of the bladder. Controls were excluded if they had a current malignancy or a history of malignancy. The outline of the study and flowchart for patient inclusion are shown in Fig. 1. DNA methylation analysis was performed in 108 patients with bladder cancer and 100 controls who were matched based on age and gender (Table 1). Of the patients with bladder cancer, $70 \%$ had a primary tumour and the disease was non-muscle invasive in $79 \%$. Histological tissues were graded following the World Health Organization (WHO) 1973 (grades 1-3 [G1-G3]) and 2004/2016 (LGHG) classification systems. Controls consisted of patients who were evaluated for haematuria $(n=34)$, patients who were diagnosed with other benign urological conditions $(n=43)$ and healthy controls $(n=23)$. The study was conducted in accordance with the Declaration of Helsinki, and the study protocol was approved by the Medical Ethics Committee (urology: 2018.355 [16-10-2018], WO 18.155 [21-12-2018]; healthy controls: 2018.657 [07-022019]). All participants gave informed written consent for study participation prior to inclusion.

\section{Urine samples}

Patient samples were collected before cystoscopy or TURBT and were processed within 24-72 $\mathrm{h}$ after collection. DNA quality was preserved by the addition of $0.6 \mathrm{M}$ ethylenediaminetetraacetic acid in a final concentration of $40 \mathrm{mM}$ [11]. Urine samples were pelleted by centrifugation of $15 \mathrm{~mL}$ urine at $800 \times g$ for $10 \mathrm{~min}$, and thereafter stored at $-20^{\circ} \mathrm{C}$. 


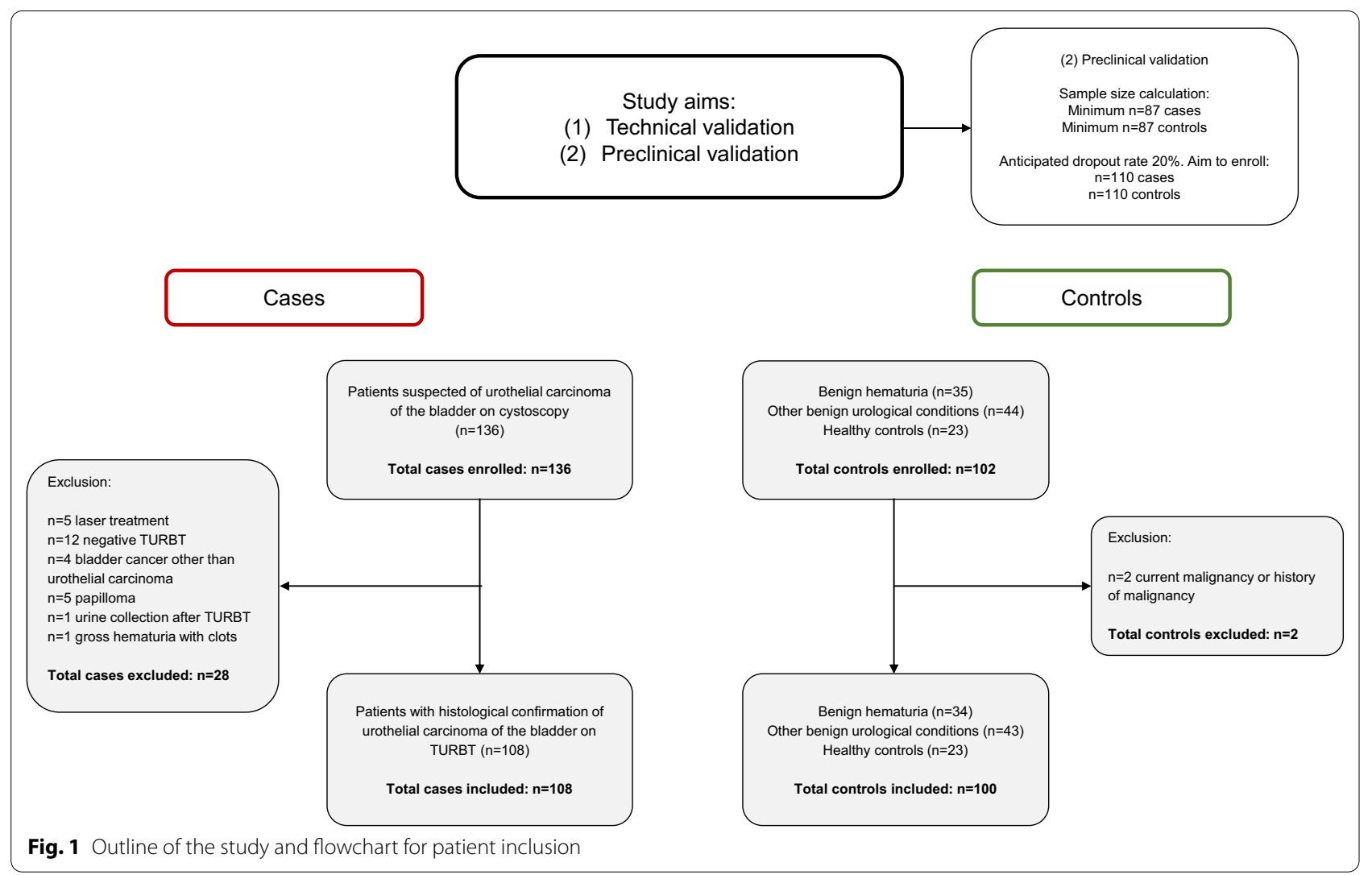

\section{DNA isolation, bisulphite conversion and quantitative methylation-specific PCR}

DNA isolation was performed with QIAamp DNA Mini Kit (Qiagen GmbH, Hilden, Germany). DNA concentrations were measured with NanoDrop 1000 (ThermoFisher Scientific, Waltham, MA, US). Next, bisulphite conversion was achieved with EZ DNA Methylation $^{\mathrm{TM}}$ Kit (Zymo Research, Orange, CA, USA). DNA isolation and bisulphite conversion were in accordance with the manufacturer's protocols. Quantitative methylation-specific PCR (qMSP) was conducted for the target genes in three different multiplex assays (FAM19A4/PHACTR3/PRDM14/ACTB; GHSR/ SST/ZIC1/ACTB; MAL/miR-129/miR-935/ACTB) as described previously $[9,12]$. qMSPs were performed with $50 \mathrm{ng}$ of bisulphite-converted DNA as input. The methylation values of the target genes were normalized for the reference gene $A C T B$ by using the comparative $\mathrm{Ct}$ method $\left(2^{-\Delta \mathrm{CT}} \times 100\right)$ to obtain $\mathrm{Ct}$ ratios. In six patients (2.9\%; one bladder cancer patient, five controls), results of all target genes from one or more multiplexes were considered invalid due to an $A C T B C t>32$ [13].

\section{Statistical analyses}

For the sample size calculation, the area under the curve (AUC) of the marker panel GHSR/MAL from the previous exploratory study was used (AUC 0.89) [9]. To obtain a maximum width of 0.1 for the two-sided $95 \%$ confidence interval (CI), a minimum sample size of $n=87$ patients with bladder cancer and $n=87$ controls was required. As a dropout rate of $20 \%$ was anticipated for cases and controls, we aimed to enrol $n=110$ participants in both groups (Fig. 1).

Categorical data were described with frequencies and percentages, and continuous data with medians and interquartile range (IQR). The Chi-square test was performed to compare categorical data between patients with bladder cancer and controls. The Mann-Whitney $U$ test was used to compare means of continuous data between both groups. The methylation levels of the urinary markers were calculated as log2-transformed $\mathrm{Ct}$ ratios and were visualized as boxplots. The Kruskal-Wallis test was used to compare the methylation levels of the urinary markers between controls with benign haematuria, other benign urological conditions and healthy controls. Since the methylation levels did not differ between 
Table 1 Baseline characteristics of the study population

\begin{tabular}{|c|c|c|c|}
\hline Characteristics & Bladder cancer ( $n=108)$ & Controls $(n=100)$ & $p$ value \\
\hline Age, $y r,(I Q R)$ & $71(63-75)$ & $66(59-75)$ & 0.25 \\
\hline Gender, $n(\%)$ & & & 0.12 \\
\hline Male & $79(73)$ & $63(63)$ & \\
\hline Female & $29(27)$ & $37(37)$ & \\
\hline \multicolumn{4}{|l|}{ WHO 1793, n (\%) } \\
\hline G1 & $17(16)$ & - & \\
\hline G2 & $39(36)$ & - & \\
\hline G3 & $52(48)$ & - & \\
\hline \multicolumn{4}{|l|}{ WHO 2004/2016, n (\%) } \\
\hline LG & $45(42)$ & - & \\
\hline $\mathrm{HG}$ & $63(58)$ & - & \\
\hline \multicolumn{4}{|l|}{ Tumour stage, n (\%) } \\
\hline $\mathrm{Ta}$ & $59(55)$ & - & \\
\hline $\mathrm{T} 1$ & $16(15)$ & - & \\
\hline Tis & $10(9)$ & - & \\
\hline$\geq T 2$ & $23(21)$ & - & \\
\hline \multicolumn{4}{|l|}{ Primary/recurrence, $n$ (\%) } \\
\hline Primary & $76(70)$ & - & \\
\hline Recurrence & $32(30)$ & - & \\
\hline \multicolumn{4}{|l|}{ Number of tumours, $n$ (\%) } \\
\hline Solitary & $59(55)$ & - & \\
\hline Multiple & $49(45)$ & - & \\
\hline \multicolumn{4}{|l|}{ Tumour size, $n(\%)$} \\
\hline$<3 \mathrm{~cm}$ & $55(51)$ & - & \\
\hline$\geq 3 \mathrm{~cm}$ & $43(40)$ & - & \\
\hline Unknown & $10(9)$ & - & \\
\hline \multicolumn{4}{|l|}{ Concomitant CIS, n (\%) } \\
\hline No & $97(90)$ & - & \\
\hline Yes & $11(10)$ & - & \\
\hline \multicolumn{4}{|l|}{ Controls, n (\%) } \\
\hline Benign haematuria & - & $34(34)$ & \\
\hline Other benign urological conditions & - & $43(43)$ & \\
\hline Healthy controls & - & $23(23)$ & \\
\hline
\end{tabular}

CIS carcinoma in situ, G grade, HG high-grade, IQR Interquartile range, LG low-grade, WHO World Health organization, yr year

the control cohorts, all 100 controls were considered as one single group. The $\mathrm{Ct}$ ratios of the urinary methylation markers were used to plot a receiver operating characteristic (ROC) curve and to establish the AUC. The level of discrimination of an AUC of 0.5-0.6 was considered very poor, $0.6-0.7$ poor, $0.7-0.8$ fair, $0.8-0.9$ good, and $0.9-1.0$ excellent [14].

The diagnostic performance of the marker panel GHSR/MAL was scored positive if at least one of both markers was positive ('believe-the-positive') $[9,15]$. Due to the use of urine pellet instead of full void urine [9], new thresholds were determined for the marker panel GHSR/ $M A L$ with Youden's J index (Additional file 1: Table S1)
[16, 17]. Leave-one-out cross-validation (LOOCV) was used to evaluate the accuracy of the diagnostic performance of the marker panel GHSR/MAL. In addition, a random forest analysis was performed to further establish the ability of all nine markers to distinguish between cases and controls.

The sensitivity of the marker panel GHSR/MAL was also determined with respect to tumour grade (G3 vs. G1-G2, and HG vs. LG), tumour stage ( $\geq \mathrm{T} 2$ vs. Ta/T1/ Tis), disease status (primary vs. recurrent) and gender (male vs. female). The Chi-square test was used to assess the differences in diagnostic performance between these subgroups of patients with bladder cancer. Based on the 
previous and current differences in the sensitivity of the marker panel GHSR/MAL for gender, post hoc analyses were performed with males and females considered as separate cohorts [9]. New thresholds of the Ct ratios were determined for both genders with Youden's J index (Additional file 1: Table S1) [16, 17].

Sample size calculation was performed with PASS version 15.0. Statistical analyses were done with SPSS Software (SPSS 26.0, IBM Corp., NY, USA) and R Statistical Software (version 3.6.1; R Foundation for Statistical Computing, Vienna, Austria). Graphs were created with GraphPad Software (GraphPad Prism 8.2.1, San
Diego, California, USA). Bonferroni correction was used to account for multiple testing. Reported $p$ values are two-sided and were considered statistically significant at $p<0.05$.

\section{Results}

\section{Technical validation}

The urinary methylation levels of FAM19A4, GHSR, MAL, miR-129, miR-935, PHACTR3, PRDM14, SST and ZIC1 were significantly higher in patients with bladder cancer than in controls (all, $p<0.001$ ) (Fig. 2). To confirm the technical performance of DNA methylation analysis

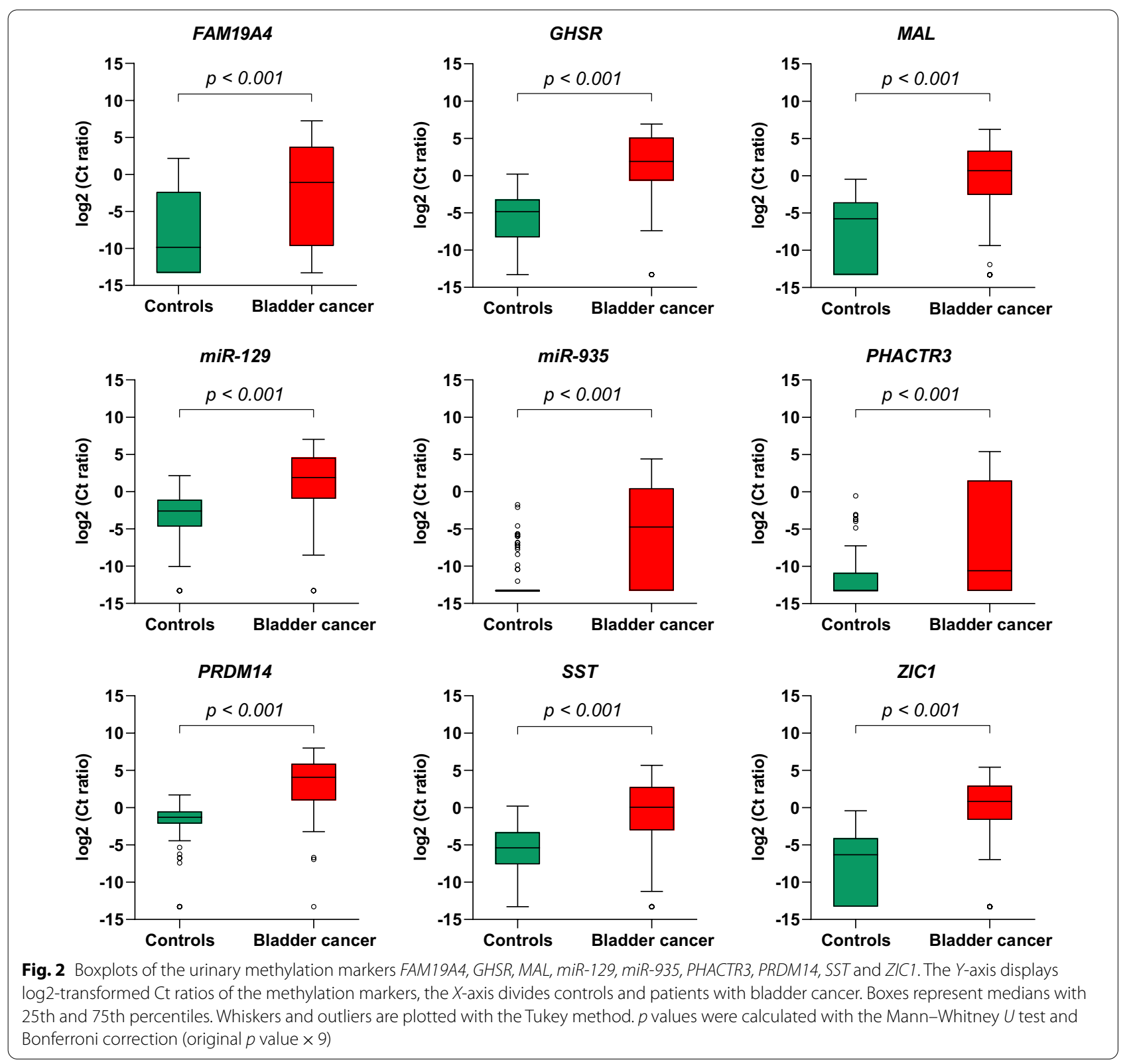


in urine pellet, we compared the AUCs of these nine methylation markers in urine pellet in this independent study population ( $n=208$ participants) to the AUCs of our previous exploratory cohort in which full void urine was used ( $n=147$ participants) [9]. Figure 3 shows a high similarity between the ROC curves of both studies [9]. All methylation markers, except $M A L$, reached a slightly higher AUC in the present study. The AUC of
ZIC1 increased most, from 0.77 in the exploratory study to 0.88 in the present study.

\section{Preclinical validation}

Next, the diagnostic performance of the previously determined optimal marker panel GHSR/MAL was assessed [9]. The marker panel reached an AUC of 0.89 (95\% CI $0.84-0.94)$, corresponding to a sensitivity of $80 \%$ (95\% CI

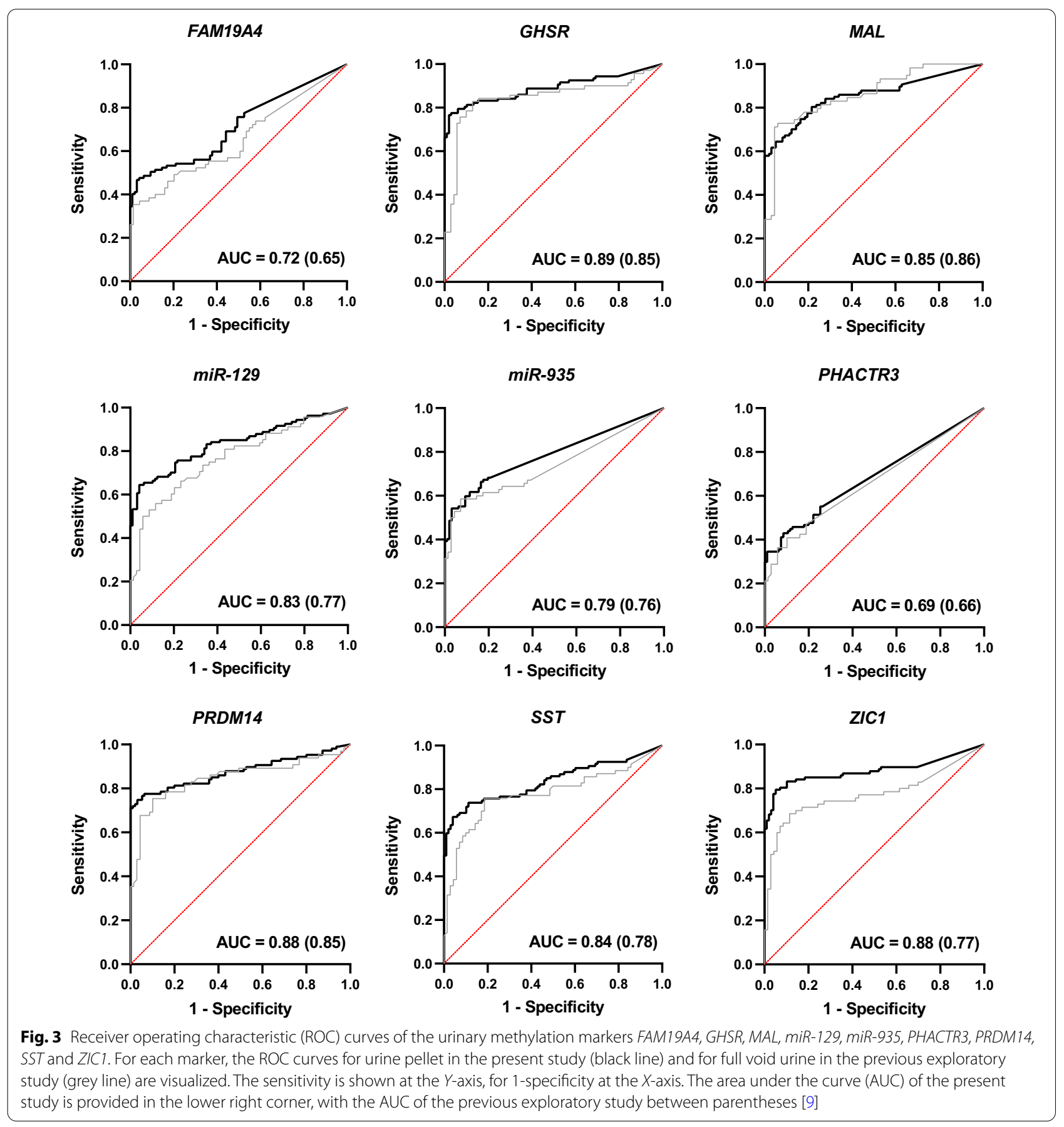


Table 2 Preclinical validation and leave-one-out cross-validation (LOOCV) of the marker panel GHSR/MAL

\begin{tabular}{lll}
\hline Study population & $\begin{array}{l}\text { GHSR/MAL } \\
\text { Preclinical validation }\end{array}$ & $\begin{array}{l}\text { GHSR/MAL } \\
\text { LOOCV }\end{array}$ \\
\hline All patients & & \\
AUC & $0.89(95 \% \mathrm{Cl} 0.84-0.94)$ & 0.89 \\
Sensitivity & $0.80(95 \% \mathrm{Cl} 0.71-0.87)$ & 0.79 \\
Specificity & $0.93(95 \% \mathrm{Cl} 0.85-0.97)$ & 0.76 \\
Males & $0.95(95 \% \mathrm{Cl} 0.92-0.99)$ & 0.95 \\
AUC & $0.92(95 \% \mathrm{Cl} 0.83-0.97)$ & 0.90 \\
Sensitivity & $0.93(95 \% \mathrm{Cl} 0.83-0.98)$ & 0.89 \\
Specificity & & \\
Females & $0.81(95 \% \mathrm{Cl} 0.70-0.92)$ & 0.81 \\
AUC & $0.79(95 \% \mathrm{Cl} 0.60-0.91)$ & 0.76 \\
Sensitivity & $0.72(95 \% \mathrm{Cl} 0.55-0.85)$ & 0.69 \\
Specificity &
\end{tabular}

AUC area under the curve, $\mathrm{Cl}$ confidence interval, LOOCV leave-one-out crossvalidation

71-87) and a specificity of 93\% (95\% CI 85-97) (Table 2). Upon LOOCV, an identical diagnostic accuracy (AUC 0.89 ) was obtained. A random forest analysis on all nine markers led to a comparable diagnostic performance with a sensitivity of $81 \%$ (95\% CI 74-89) and a specificity of $93 \%$ (95\% CI 88-98). This underlines the appropriate selection of GHSR/MAL as the optimal marker panel to distinguish between bladder cancer patients and controls.

Results are given for all patients, for males and for females. Based on previous and current differences in the sensitivity of the marker panel GHSR/MAL for gender, post hoc analyses were performed with males and females considered as separate cohorts. Hereto, new thresholds of the $\mathrm{Ct}$ ratios were determined with Youden's J index for males and females separately (Additional file 1: Table S1) $[16,17]$.

The sensitivity of the marker panel GHSR/MAL was determined in different subgroups of patients with bladder cancer. These subgroup analyses showed that sensitivity increased with higher tumour grades (G3 vs. G1-G2, HG vs. LG), higher tumour stages ( $\geq \mathrm{T} 2 \mathrm{vs.} \mathrm{Ta/}$ $\mathrm{T} 1 /$ Tis), in primary vs. recurrent tumours, and in males vs. females (Table 3). Sensitivity of the marker panel GHSR/MAL was $59 \%$ in females and $89 \%$ in males $(p=0.001)$.

Differences in sensitivity between the dichotomized subgroups were analysed with the Chi-square test.

\section{Post hoc analyses for gender}

As the sensitivity of the marker panel GHSR/MAL differed substantially between males and females, its diagnostic performance was subsequently assessed with new thresholds for both genders separately (Additional file 1:
Table 3 Sensitivity of the marker panel GHSR/MAL in different subgroups of patients with bladder cancer

\begin{tabular}{llc}
\hline Subgroups & GHSR/MAL & p value \\
\hline $\begin{array}{l}\text { All }(n=108) \\
\text { WHO 1973 }\end{array}$ & $80(71-87)$ & $<0.001$ \\
G1-G2 $(n=56)$ & $66(52-78)$ & \\
G3 $(n=52)$ & $96(85-99)$ & $<0.001$ \\
WHO 2004/2016 & & \\
LG $(n=45)$ & $58(42-72)$ & \\
HG $(n=63)$ & $97(88-99)$ & \\
Tumour stage & & \\
Ta/T1/Tis $(n=85)$ & $75(64-84)$ & $<0.007$ \\
$\geq T 2$ ( $n=23)$ & $100(82-100)$ & \\
Primary/recurrence & & \\
Primary $(n=76)$ & $89(80-95)$ & \\
Recurrence $(n=32)$ & $59(41-76)$ & \\
Gender & & \\
Male $(n=79)$ & $89(79-94)$ & \\
Female $(n=29)$ & $59(39-76)$ & \\
\hline
\end{tabular}

$H G$ high-grade, $G$ grade, $L G$ low-grade

Table S1). In males, the marker panel reached an AUC of 0.95 (95\% CI $0.92-0.99$ ), corresponding to a sensitivity of $92 \%$ (95\% CI 83-97) and a specificity of $93 \%$ (95\% CI 83-98) (Table 2). For females, the marker panel yielded an AUC of 0.81 (95\% CI $0.70-0.92$ ), at a sensitivity of $79 \%$ (95\% CI 60-91) and a specificity of 72\% (95\% CI 55-85). LOOCV reached a similar diagnostic accuracy in both genders (Table 2).

\section{Discussion}

Technical validation of DNA methylation analysis showed that its application to either urine pellet or full void urine is suitable for urinary bladder cancer detection. Highly reproducible results were found between both urine fractions in independent study populations. The robustness of DNA methylation analysis is further supported by preclinical validation of the marker panel GHSR/MAL. A cross-validated AUC of 0.89 was obtained, which is consistent with the previous exploratory study [9]. A random forest analysis confirmed that GHSR/MAL was rightfully selected as the optimal marker panel to differentiate cases from controls. The marker panel GHSR/MAL did not miss any of the muscle-invasive tumours and only two of the non-invasive HG/G3 tumours. Present findings underline the potential of this methylation test for future bladder cancer diagnostics.

In general, for clinical adaption and prevention of unnecessary cystoscopies, urinary biomarker tests should be highly sensitive and specific. For the detection of primary disease, a high sensitivity of urinary biomarker 
tests is of utmost importance, as the consequences of not detecting a clinically significant bladder cancer may be high. When used in follow-up for recurrence, it might be assumed that a lower sensitivity is acceptable when urinary biomarker tests are alternated with cystoscopies, since an undetected bladder cancer in urine is then noticed on the next cystoscopic evaluation. Of course, the delayed detection of a bladder tumour is less critical in case of low-risk disease compared to high-risk disease. An alternating approach of cystoscopies and urinary biomarker tests could reduce the number of hospital visits and invasive cystoscopies. It is expected that besides improving quality of life of patients under follow-up for recurrence, this approach will also reduce the economic burden associated with bladder cancer.

Our and other urinary biomarker tests performed better in higher tumour grades and stages [18-23]. Four large-scale publications concerning the Bladder EpiCheck test, encompassing 15 undisclosed methylation markers for the surveillance of NMIBC patients, showed that overall sensitivity and specificity ranged $62-68 \%$ and $82-88 \%$, respectively [20-23]. However, the sensitivity was higher in HG tumours (79-89\%) compared to LG tumours (40-54\%). In addition, one of these studies reported that the sensitivity decreased from $100 \%$ in $\mathrm{T} 1$ tumours to $52 \%$ in Ta tumours [23]. The sensitivity of urinary biomarker tests is presumably lower in patients with favourable tumour characteristics (small, LG or low-stage tumours) as these tumours harbour less molecular abnormalities [24, 25]. Diagnostic performance of the marker panel GHSR/MAL was also higher in primary than in recurrent tumours. It is expected that bladder tumours have a higher tumour load at primary diagnosis than during surveillance, resulting in a higher sensitivity of the marker panel GHSR/MAL in primary tumours. A combined assay of complementary biomarker types might encompass the variability in tumour characteristics [6]. Recently, a prospective study in 838 haematuria patients used a six-marker model consisting of three methylation markers (ONECUT2, OTX1 and TWIST1) and three mutation markers (FGFR3, HRAS and TERT), as well as the presence of macroscopic haematuria. The combined assay reached $96 \%$ sensitivity at $73 \%$ specificity for the detection of primary bladder cancer, with $91 \%$ sensitivity in LG and 99\% sensitivity in HG tumours [26]. Moreover, technological developments that enable the sensitive detection of low amounts of tumour-related material may further increase the diagnostic performance of urinary biomarker tests in all bladder tumours.

Similar to previous studies we found a marked difference between the sensitivity of the marker panel GHSR/ $M A L$ in males and females, whereas its specificity was similar in both genders $[9,18,27]$. The marker panel performed excellent in males, while its diagnostic performance was good in females. We assume that methylated DNA in urine of females is diluted due to the abundance of unmethylated DNA from normal cells of gynaecological origin [18, 27]. Furthermore, the presence of leucocytes might also cause high background values of unmethylated DNA in urine of females [27, 28]. A possible method to enhance the sensitivity of the marker panel GHSR/MAL in females comprises the use of midstream urine, as gynaecological cells are predominantly present in the first void [29]. This approach might prove to be particularly beneficial in female patients with favourable tumour characteristics, in whom the amount of tumourrelated DNA in urine is expected to be low [30]. Another possible method to overcome gender-related differences is the use of different thresholds for males and females (Table 2 and Additional file 1: Table S1). The present study was, however, limited by the low number of female participants, hampering any definitive conclusions on this topic.

Other limitations of this study concern its case-control design, which hampers an evaluation of the diagnostic value of the marker panel GHSR/MAL in routine clinical practice. Secondly, patients with primary as well as recurrent bladder cancer were included. Thirdly, controls were matched based on age and gender, but not all visited the urology clinic because of bladder cancer symptoms. Although methylation levels did not differ between the control cohorts, specificity might still be overestimated.

Taken together, testing for the methylation marker panel GHSR/MAL in urine may provide a valuable noninvasive strategy to detect bladder cancer. Present findings warrant further studies on the clinical value of this methylation test for the primary detection of bladder cancer and in the follow-up of NMIBC patients after TURBT, as a means to reduce the number of invasive cystoscopies.

\section{Conclusions}

Present data support the technical robustness of DNA methylation in urine by demonstrating the suitability of both urine pellet and full void urine for the non-invasive detection of bladder cancer. Preclinical validation of the marker panel GHSR/MAL yielded a cross-validated AUC of 0.89 , which is identical to a previous exploratory study.

\section{Supplementary Information}

The online version contains supplementary material available at https://doi. org/10.1186/s13148-022-01240-8.

Additional file 1: Table S1. Thresholds of the Ct ratios for the marker panel GHSR/MAL in urine pellet, determined by Youden's J index. 


\section{Acknowledgements}

The authors would like to thank A.P. van Splunter for her technical support, the URIC team and the UROTECT study team for the collection of urine samples, and the Department of Pathology of OLVG for the revision of the tissue slides.

\section{Authors' contributions}

AH: Conceptualization, Methodology, Validation, Formal Analysis, Investigation, Resources, Data Curation, Writing—Original Draft, Writing—Review and Editing, Visualization, Project Administration; IB: Writing-Original Draft, Writing-Review and Editing; JB: Conceptualization, Writing-Review and Editing; PK: Investigation, Writing - Review and Editing, Supervision; AV: Writing — Review and Editing, Supervision; BW: Methodology, Validation, Formal Analysis; RvM: Resources, Writing-Review and Editing, Supervision; RS: Conceptualization, Methodology, Investigation, Resources, Data Curation, Writing —Original Draft, Writing — Review and Editing, Visualization, Supervision, Funding Acquisition; JN: Conceptualization, Methodology, Investigation, Resources, Writing — Original Draft, Writing — Review and Editing, Visualization, Supervision, Funding Acquisition. All authors have read and approved the fina version of manuscript.

\section{Funding}

This work was supported by the Weijerhorst Foundation.

\section{Availability of data and materials}

Data are available upon reasonable request

\section{Declarations}

Ethics approval and consent to participate

The study was conducted in accordance with the Declaration of Helsinki, and the study protocol was approved by the Medical Ethics Committee (urology: 2018.355 [16-10-2018], WO 18.155 [21-12-2018]; healthy controls: 2018.657 [07-02-2019]). All participants gave informed written consent for study participation prior to inclusion.

\section{Consent for publication}

Not applicable.

\section{Competing interests}

R.D.M. Steenbergen has a minority share in Self-screen BV, a spin-off company of Amsterdam UMC, location VUmc. J. Bosschieter, J.A. Nieuwenhuijzen and R.D.M. Steenbergen are inventors on patents related to the work.

\section{Author details}

'Department of Urology, Amsterdam UMC, Vrije Universiteit Amsterdam, Amsterdam, The Netherlands. ${ }^{2}$ Department of Pathology, Cancer Center Amsterdam, Amsterdam UMC, Vrije Universiteit Amsterdam, Amsterdam, The Netherlands. ${ }^{3}$ Department of Urology, OLVG, Amsterdam, The Netherlands. ${ }^{4}$ Epidemiology and Data Science, Amsterdam UMC, Vrije Universiteit Amsterdam, Amsterdam, the Netherlands.

Received: 9 November 2021 Accepted: 26 January 2022 Published online: 05 February 2022

\section{References}

1. DeGeorge KC, Holt HR, Hodges SC. Bladder cancer: diagnosis and management of bladder cancer. BJU Int. 2017;120(6):755-65.

2. Svatek RS, et al. The economics of bladder cancer: costs and considerations of caring for this disease. Eur Urol. 2014;66(2):253-62

3. Yafi FA, et al. Prospective analysis of sensitivity and specificity of urinary cytology and other urinary biomarkers for bladder cancer. Urol Oncol. 2015;33(2):66e25-31.

4. Raitanen MP, et al. Differences between local and review urinary cytology in diagnosis of bladder cancer. An interobserver multicenter analysis. Eur Urol. 2002;41(3):284-9.

5. Miyake $M$, et al. Emerging biomarkers for the diagnosis and monitoring of urothelial carcinoma. Res Rep Urol. 2018;10:251-61.
6. Tan WS, et al. Novel urinary biomarkers for the detection of bladder cancer: a systematic review. Cancer Treat Rev. 2018;69:39-52.

7. Bosschieter J, et al. The diagnostic accuracy of methylation markers in urine for the detection of bladder cancer: a systematic review. Epigenomics. 2018;10(5):673-87.

8. Kandimalla R, van Tilborg AA, Zwarthoff EC. DNA methylation-based biomarkers in bladder cancer. Nat Rev Urol. 2013;10(6):327-35.

9. Bosschieter J, et al. A two-gene methylation signature for the diagnosis of bladder cancer in urine. Epigenomics. 2019:11(3):337-47.

10. Hentschel $A E$, et al. Comparative analysis of urine fractions for optimal bladder cancer detection using DNA methylation markers. Cancers (Basel). 2020;12(4):859.

11. Bosschieter J, et al. A protocol for urine collection and storage prior to DNA methylation analysis. PLoS ONE. 2018;13(8):e0200906.

12. Verlaat W, et al. Genome-wide DNA methylation profiling reveals methylation markers associated with $3 q$ gain for detection of cervical precancer and cancer. Clin Cancer Res. 2017;23(14):3813-22.

13. Schmittgen TD, Livak KJ. Analyzing real-time PCR data by the comparative C(T) method. Nat Protoc. 2008;3(6):1101-8.

14. Simundic AM. Measures of diagnostic accuracy: basic definitions. EJIFCC. 2009;19(4):203-11.

15. Marshall R. The predictive value of simple rules for combining two diagnostic tests. Biometrics. 1989;45:1213-22.

16. Schisterman EF, et al. Optimal cut-point and its corresponding Youden Index to discriminate individuals using pooled blood samples. Epidemiology. 2005;16(1):73-81.

17. Youden WJ. Index for rating diagnostic tests. Cancer. 1950;3(1):32-5.

18. Beukers W, et al. FGFR3, TERT and OTX1 as a urinary biomarker combination for surveillance of patients with bladder cancer in a large prospective multicenter study. J Urol. 2017;197(6):1410-8.

19. Dahmcke $C M$, et al. A prospective blinded evaluation of urine-DNA testing for detection of urothelial bladder carcinoma in patients with gross hematuria. Eur Urol. 2016;70(6):916-9.

20. D'Andrea D, et al. Diagnostic accuracy, clinical utility and influence on decision-making of a methylation urine biomarker test in the surveillance of non-muscle-invasive bladder cancer. BJU Int. 2019;123(6):959-67.

21. Trenti $\mathrm{E}$, et al. Diagnostic predictive value of the Bladder EpiCheck test in the follow-up of patients with non-muscle-invasive bladder cancer. Cancer Cytopathol. 2019;127(7):465-9.

22. Trenti $\mathrm{E}$, et al. Comparison of 2 new real-time polymerase chain reactionbased urinary markers in the follow-up of patients with non-muscle-invasive bladder cancer. Cancer Cytopathol. 2020;128(5):341-7.

23. Witjes JA, et al. Performance of the bladder EpiCheck methylation test for patients under surveillance for non-muscle-invasive bladder cancer: results of a multicenter, prospective. Blind Clin Trial Eur Urol Oncol. 2018;1(4):307-13.

24. Knowles MA, Hurst CD. Molecular biology of bladder cancer: new insights into pathogenesis and clinical diversity. Nat Rev Cancer. 2015;15(1):25-41.

25. Liedberg F, et al. The importance of being grade 3: WHO 1999 versus WHO 2004 pathologic grading. Eur Urol. 2012;62(4):620-3.

26. van Kessel KEM, et al. A urine based genomic assay to triage patients with hematuria for cystoscopy. J Urol. 2020;204(1):50-7.

27. Kohler CU, et al. Noninvasive diagnosis of urothelial cancer in urine using DNA hypermethylation signatures-Gender matters. Int J Cancer. 2019;145(10):2861-72

28. De Strooper LM, et al. CADM1, MAL and miR124-2 methylation analysis in cervical scrapes to detect cervical and endometrial cancer. J Clin Pathol. 2014;67(12):1067-71.

29. Vorsters A, et al. Optimization of HPV DNA detection in urine by improving collection, storage, and extraction. Eur J Clin Microbiol Infect Dis. 2014;33(11):2005-14.

30. Larsen LK, et al. DNA-methylation-based detection of urological cancer in urine: overview of biomarkers and considerations on biomarker design, source of DNA, and detection technologies. Int J Mol Sci. 2019;20(11):2657.

\section{Publisher's Note}

Springer Nature remains neutral with regard to jurisdictional claims in published maps and institutional affiliations. 